parameters were compared using an independent samples ttest.

Results The mean follow-up was $32.8 \pm 2.5$ months. A standard 16 -gene panel was performed in all patients. Fourteen patients (54\%) were G+ve (4 MYBPC3, 3 M7H7 and 7 others). G +ve patients were more likely to be male (57\%), younger (39 vs 66 years, $\mathrm{p}=0.0003)$ and have a family history of HCM (43 vs $8 \%, p=0.048$ ). There was no difference in baseline diastolic septal diameter ( $\mathrm{G}+\mathrm{ve} 16.1 \pm 5.3 \mathrm{~mm}$ vs $\mathrm{G}$-ve 17.4 $\pm 7.3 \mathrm{~mm}, \mathrm{p}=0.6)$ or ejection fraction $(\mathrm{G}+\mathrm{ve} 64 \pm 2 \%$ vs $\mathrm{G}$-ve $63 \pm 2 \%, \mathrm{p}=0.697)$.

TDI parameters during follow up revealed the magnitude of deterioration in medial $\mathrm{E}$, medial $\mathrm{E} / \mathrm{E}$, lateral $\mathrm{E}$ and lateral $\mathrm{E} / \mathrm{E}$ was greater in $\mathrm{G}$-ve compared to $\mathrm{G}+\mathrm{ve}$ patients. There was also a trend for a greater decrease in medial and lateral $S$ in $\mathrm{G}$-ve patients.

During follow up we did not observe any significant differences in the change of LA diameter $(\mathrm{G}+\mathrm{ve}+3.29( \pm 6.29)$ vs $\mathrm{G}$-ve+3.08( \pm 5.50$), \quad \mathrm{p}=0.932)$ or $\mathrm{LV} \max$ wall thickness $(\mathrm{G}+\mathrm{ve}-1.07 \pm 5.05$ vs $\mathrm{G}-\mathrm{ve}+0.20 \pm 2.61, \mathrm{p}=0.440)$. Overall changes in sudden cardiac death (SCD) risk scores remained similar $(\mathrm{G}+\mathrm{ve}+0.27( \pm 0.48)$ vs $\mathrm{G}$-ve $+0.75( \pm 0.64), \mathrm{p}=0.378)$. There were no sudden cardiac deaths or shockable events.

Conclusion In this single-centre HCM clinic, despite no change in SCD risk scores or measurements of cardiac morphology, G-ve patients have a greater rate of deterioration of TDI parameters with a significant reduction in diastolic filling and a trend towards reduction in longitudinal systolic function. Although G-ve patients were older than $\mathrm{G}+\mathrm{ve}$, the rate of decline in TDI velocities over a 33 month period is greater than what would be expected in the normal ageing population.

\section{RELATIONSHIP BETWEEN PLASMA CONCENTRATIONS OF B-TYPE NATRIURETIC PEPTIDE AND EXERCISE CAPACITY IN HYPERTROPHIC CARDIOMYOPATHY}

${ }^{1}$ Amrit Lota, ${ }^{2}$ Florence Mouy*, ${ }^{2}$ Rebecca Wassall, ${ }^{3}$ Simon Newsome, ${ }^{1}$ Brian Halliday, ${ }^{2} J o h n$ Baksi, ${ }^{2}$ Antonis Pantazis, 'James Ware, ${ }^{2}$ Stuart Cook, 'Dudley Pennell, ${ }^{2} J o h n$ Cleland, ${ }^{1}$ Sanjay Prasad. ${ }^{1}$ Royal Brompton Hospital, NHLl; ${ }^{2}$ Royal Brompton Hospital; ${ }^{3}$ London School of Hygiene and Tropical Medicine

10.1136/heartjnl-2017-311726.126
Background Hypertrophic cardiomyopathy (HCM) is characterised by increased left ventricular wall thickness leading to exercise intolerance and heart failure. Peak oxygen consumption (peak VO2) on cardiopulmonary exercise testing (CPET) is often used as a functional marker alongside B-type natriuretic peptide (BNP). However, CPET is complex and time-consuming, access may be limited, and peak VO2 can be influenced by physical deconditioning and motivation. Our aims were to determine the association between BNP and submaximal exercise parameters, ventilatory efficiency (VE/VCO2) and anaerobic threshold; and to determine whether $f$ these were effective surrogate markers of functional status in patients with HCM.

Methods We retrospectively reviewed data for all patients with HCM in our local cardiomyopathy service. A diagnosis of HCM was based on LV hypertrophy $>15 \mathrm{~mm}$ as per current ESC guidelines. Patients underwent contemporaneous echocardiography, CPET using maximal treadmill ergometry and BNP measurement at sequential annual clinic visits $[1$ (18\%), 2 (52\%), $3(20 \%)$ or $\geq 4$ visits (10\%)] yielding data from 252 observations over 8 years for 119 individual patients. Univariable and multivariable linear regression was used to investigate variables associated with the results of CPET. Robust standard errors were used to correct for multiple observations from the same patients.

Results In our cohort of 119 patients (mean age $49 \pm 17$ years, $66 \%$ men), mean LVEF was $75 \pm 7 \%$, maximal wall thickness was $19 \pm 5 \mathrm{~mm}$, and left ventricular outflow tract obstruction was present in $15 \%$. The mean peak V02 was $26 \pm 8 \mathrm{~L} / \mathrm{min}$, mean VE/VCO2 slope was $30 \pm 6$ and median BNP was 93.5 ng/L (IQR 43.5-232.5). On univariable analysis, several markers were found to predict peak VO2; age $\left(\beta^{2}=-4.5\right.$; $95 \%$ CI $-6.1--3.0 ; \mathrm{p}<0.00001)$, male sex $\left(\beta^{2}=5.2\right.$; $95 \%$ CI $2.3-8.1 ; \mathrm{p}<0.001)$, LV ejection fraction $\left(\beta^{2}=-2.1\right.$; $95 \%$ CI $-3.6--0.6 ; \mathrm{p}<0.01), \quad \mathrm{BNP} \quad\left(\beta^{2}=-4.0 ; 95 \% \mathrm{CI}\right.$ $-5.8--2.2 ; \mathrm{p}<0.0001)$ and echocardiographic markers of diastolic dysfunction, for example, $\mathrm{E} / \mathrm{E}$ lateral ratio $\left(\beta^{2}=-3.4\right.$; $95 \% \mathrm{CI}-4.8--2.0 ; \mathrm{p}<0.00001)$. Predictors of $\mathrm{VE} / \mathrm{VCO} 2$ were age $\left(\beta^{2}=1.5 ; 95 \%\right.$ CI $\left.0.6-2.3 ; \mathrm{p}<0.01\right)$, BNP $\left(\beta^{2}=2.4\right.$; $95 \%$ CI $1.5-3.4 ; \mathrm{p}<0.00001)$ and similar echocardiographic markers of diastolic dysfunction. Multivariable analysis revealed that BNP was associated with peak VO2 $\left(\beta^{2}=-2.0\right.$; $95 \%$ CI $-3.0--1.1 ; \mathrm{p}<0.0001)$ but also age, male sex,

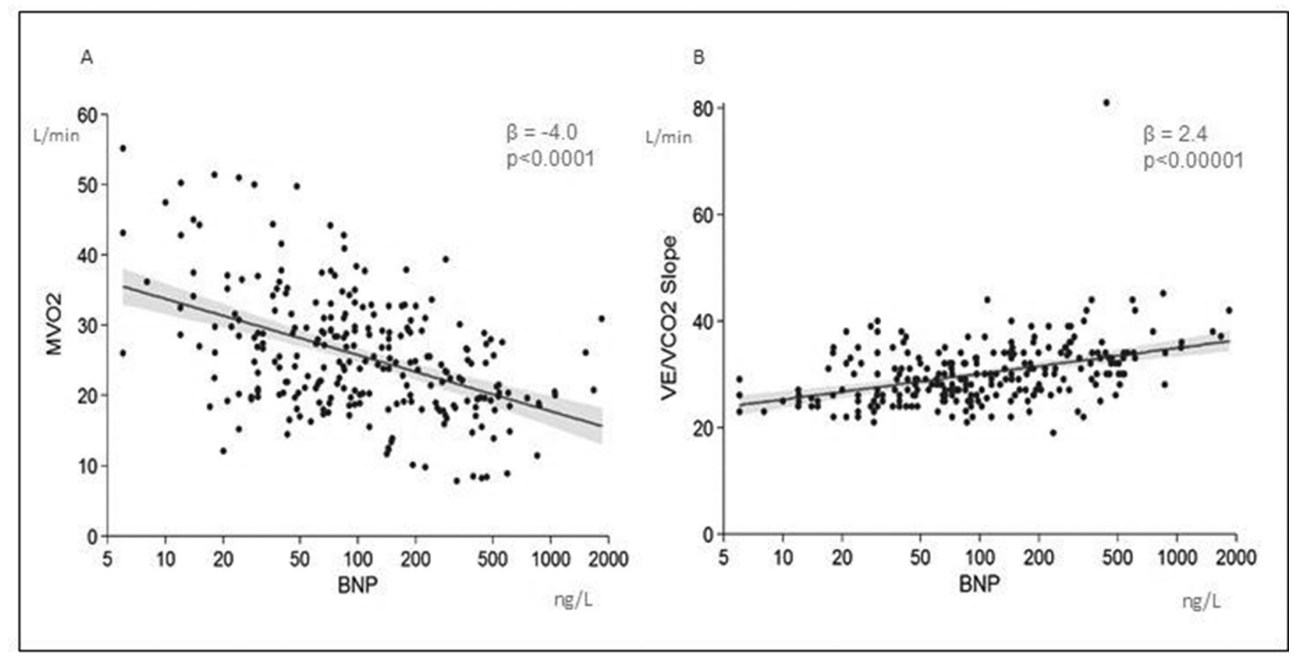

Abstract 127 Figure 1 Association between $\mathrm{BNP}$ and peak $\mathrm{VO}_{2}(\mathrm{~A})$ and $\mathrm{VE} / \mathrm{VCO}_{2}(\mathrm{~B})$ inpatients with $\mathrm{HCM}$. 
indexed RV end-diastolic volume and body weight, whilst BNP was independently associated with VE/VCO2 $\left(\beta^{2}=0.8 ; 95 \% \mathrm{CI}\right.$ $0.1-1.6 ; \mathrm{p}<0.001)$, and to a lesser extent, anaerobic threshold $\left(\beta^{2}=-1.4 ; 95 \%\right.$ CI $\left.-2.2--0.6 ; p=0.001\right)$.

Conclusion In this cohort, BNP is independently associated with VE/VCO2, a marker of ventilation-perfusion mismatch, which is known to provide a more reliable assessment of exercise intolerance irrespective of effort level. These results therefore support a role for BNP as an useful marker of submaximal exercise capacity and for consideration in guiding prognosis.

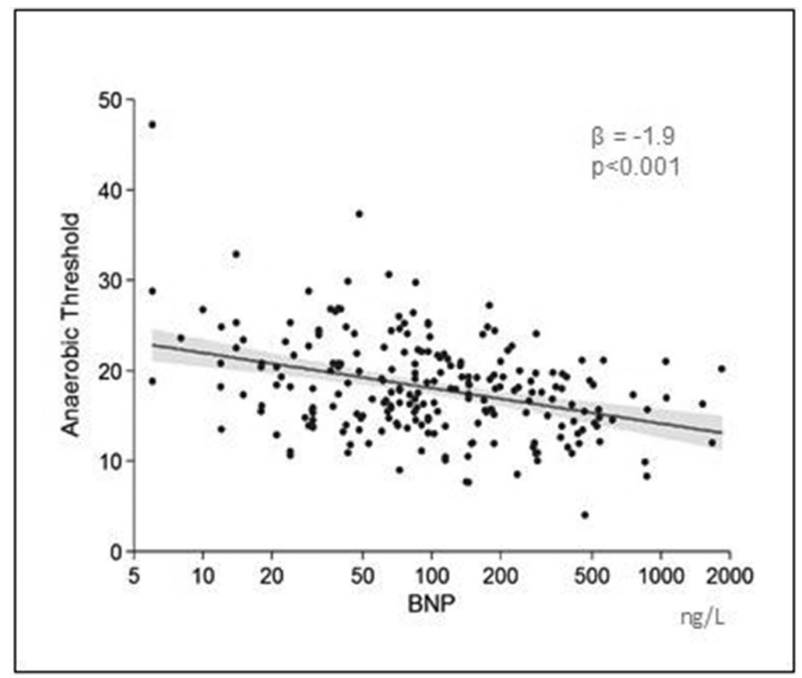

Abstract 127 Figure 2 Association between BNP and anaerobic threshold n HCM

\section{8 \\ GENOMIC INSIGHTS OF ECG STRAIN PATTEN IN AORTIC STENOSIS: T WAVE INVERSION AND ST-SEGMENT DEPRESSION ARE UNDERLINED BY DIFFERENT MOLECULAR PATHWAYS}

${ }^{1}$ Jiangting Hu*, ${ }^{2}$ John Pepper, ${ }^{1}$ Francesco Pezzella, ${ }^{3}$ Kevin Gatter, ${ }^{4} \mathrm{Xu}$ Yu Jin. ${ }^{1}$ Nuffield Division of Clinical Lab Sciences, Radcliffe Department of Medicine, University of Oxford; ${ }^{2}$ academic Cardiac Surgery, Royal Brompton Hospital; ${ }^{3}$ Nuffield Division of Clinical Lab Sciences, Radcliffe Department of Medician, University of Oxford; ${ }^{4}$ Department of Cardiothoracic Surgery, John Radcliffe Hospital

\subsection{6/heartinl-2017-311726.127}

Background ECG strain pattern is frequently presented in AS patients as a marker of LV hypertrophy and is emerging as one of the predictors for AVR. Our previous study had demonstrated that the normalisation of ST depression occurred within first 24 hours after AVR for AS while that of inverted $\mathrm{T}$ wave took $6-12$ months in parallel to the time course of $\mathrm{LVH}$ regression. We hereby studied myocardium gene expression profiling and tested the hypothesis that different molecular mechanisms could have underlined the ST depression and $\mathrm{T}$ wave inversion.

Material and methods We studied 17 AS patients with age 73 \pm 8.5 years and 12 males. LV biopsy was taken during AVR. Myocardial gene expression profiling was studied using Stanford Human Exonic Evidence Based Oligonucleotide (HEEBO) array. $\mathrm{T}$ wave inversion was defined by mean voltage of $\mathrm{T}$ wave in ECG lead I, aVL, V5 and V6 below $-50 \mathrm{uV}$ (T-invert, $\mathrm{n}=8$; T-norm, $\mathrm{n}=9$ ). ST depression was defined by a mean value of ST in the same leads being below $-50 \mathrm{uV}$ (ST-dep, $\mathrm{n}=9$; ST-norm, $\mathrm{n}=8$ ). Differential gene expression profiling within ST and T wave sub-group were carried out by parametric permutative (permutation times $=1000$ ) t-test using the $\mathrm{p}$ value cut-off 0.01 respectively. Further gene functional annotation clusters were performed using Database for Annotation, Visualisation and Integrated Discovery (DAVID, NIH) and pathway analysis of GeneSpring GX10.

Results We identified 588 differentially expressed genes (DEGs) between the T-invert and T-norm with 308 genes upregulated and 280 genes down-regulated in T-invert. Using Direct Interaction Network, genes with more intensive interactions were PLCL1, FGF1, MGP, FABP4, IL31 and IFI27 in up regulation and EGF, PAK2 SYT1, PPA1 and CSH1 in downregulation, in T-invert. Between ST-dep and ST-normal, we identified 202 DEGs, with 95 up and 107 down regulated in ST-dep. Using Direct Interaction Network analysis, we identified interactive connexion of genes, including ARHGEF1, ARRB1, PPM1A, CD28, SNX9, PRSS27, ASXL1, HDAC9 and BCOR, with no centre nodes orientation. The Significantly Enriched Core Pathways Analysis and DAVID gene functional annotation clustering further revealed that the top pathways underlined the genes of T-invert were oxidative phosphorylation, mitochondria, cell cycle/mitosis, cell adhesion, EGF/ERBB pathway, fatty acid metabolism; whilst for the genes of STdep, the main pathways were $G$ protein signalling pathway, transcription regulation of adipocyte differentiation and membrane trafficking/endocytosis.

Conclusions Our study provides new molecular insights into ECG strain pattern in LV hypertrophy due to AS. T wave inversion appears to be involved in down regulation of energy metabolism and cell cycle but ST-depression is mainly associated with changes in signalling transduction. Understanding these different biological significance will help a more precise clinical interpretation of ECG strain pattern and its changes following AVR for AS.

\section{ASSOCIATION OF ADVERSE VENTRICULAR REMODELLING AND GENDER IN AORTIC STENOSIS}

Anvesha Singh*, Daniel Chan, Leong Ng, Gerry McCann. University of Leicester

\subsection{6/heartjnl-2017-311726.128}

Introduction Previous echocardiography studies have demonstrated smaller left ventricles(LV) with more hypertrophy and concentric remodelling in females with Aortic Stenosis (AS) compared to males. However, more recent cardiac magnetic resonance(CMR) studies have shown lower LV mass and mass/ volume in females. We utilised CMR to assess myocardial perfusion and tissue characterisation, in addition to remodelling and function, and looked at biomarkers of fibrosis (Syndecan4 and MMP-3) in male and female patients.

Methods Subjects with asymptomatic moderate to severe AS ( 2 of: aortic valve area $<1.5 \mathrm{~cm}^{2}$, peak gradient $>36 \mathrm{~mm} \mathrm{Hg}$ or mean gradient $>25 \mathrm{~mm} \mathrm{Hg}$ ) were recruited in this prospective, multi-centre, observational study. All patients underwent venepuncture, echocardiogram and a comprehensive stress CMR.

Results 174 patients (133 male) were recruited. Females were slightly younger but there was no difference in resting haemodynamics, co-morbidities or AS severity between the genders (Table 1). Male patients had significantly higher LV volumes 